\title{
Brain Mechanisms Supporting the Modulation of Pain by Mindfulness Meditation
}

\author{
Fadel Zeidan, ${ }^{1}$ Katherine T. Martucci, ${ }^{1}$ Robert A. Kraft, ${ }^{2}$ Nakia S. Gordon, ${ }^{3}$ John G. McHaffie, ${ }^{1}$ and Robert C. Coghill ${ }^{1}$ \\ Departments of ${ }^{1}$ Neurobiology and Anatomy and ${ }^{2}$ Biomedical Engineering, Wake Forest University School of Medicine, Winston-Salem, North Carolina \\ 27157, and ${ }^{3}$ Psychology Department, Marquette University, Milwaukee, Wisconsin 53233
}

The subjective experience of one's environment is constructed by interactions among sensory, cognitive, and affective processes. For centuries, meditation has been thought to influence such processes by enabling a nonevaluative representation of sensory events. To better understand how meditation influences the sensory experience, we used arterial spin labeling functional magnetic resonance imaging to assess the neural mechanisms by which mindfulness meditation influences pain in healthy human participants. After $4 \mathrm{~d}$ of mindfulness meditation training, meditating in the presence of noxious stimulation significantly reduced pain unpleasantness by $57 \%$ and pain intensity ratings by $40 \%$ when compared to rest. A two-factor repeated-measures ANOVA was used to identify interactions between meditation and pain-related brain activation. Meditation reduced pain-related activation of the contralateral primary somatosensory cortex. Multiple regression analysis was used to identify brain regions associated with individual differences in the magnitude of meditation-related pain reductions. Meditation-induced reductions in pain intensity ratings were associated with increased activity in the anterior cingulate cortex and anterior insula, areas involved in the cognitive regulation of nociceptive processing. Reductions in pain unpleasantness ratings were associated with orbitofrontal cortex activation, an area implicated in reframing the contextual evaluation of sensory events. Moreover, reductions in pain unpleasantness also were associated with thalamic deactivation, which may reflect a limbic gating mechanism involved in modifying interactions between afferent input and executive-order brain areas. Together, these data indicate that meditation engages multiple brain mechanisms that alter the construction of the subjectively available pain experience from afferent information.

\section{Introduction}

Rather than a passive reflection of afferent information, our perception of the sensory environment is shaped by our own unique past experiences, current cognitive state, and future expectations. Perhaps no better example of this dynamic integrative process exists than the transformation of nociceptive information into the subjective experience of pain. Although the intrinsically salient nature of noxious stimuli initiates a host of physiological and neurophysiological alterations within our bodies, its effects are nevertheless highly malleable and subject to modification. Contemplatives have emphasized that the nature of our mental activity can be manipulated to promote self-regulation through the practice of mindfulness meditation (Nhât and Vo-Dinh, 1987). One form of mindfulness meditation is Shamatha (Wallace, 2006) or focused attention (Lutz et al., 2008). Focused attention is the cognitive practice of sustaining attention on the changing sensations of the breath, monitoring discursive events as they arise, disengaging from those events without affective

Received Nov. 3, 2010; revised Jan. 6, 2011; accepted Feb. 13, 2011.

This study was supported by the Mind and Life Institute Varela Grant, National Institutes of Health Grant NS39426, and the Center for Biomolecular Imaging of Wake Forest University School of Medicine.

The authors declare no competing financial interests.

Correspondence should be addressed to Dr. Fadel Zeidan, Department of Neurobiology and Anatomy, Wake Forest University School of Medicine, Medical Center Boulevard, Winston-Salem, NC 27157. E-mail: fzeidan@wfubmc.edu.

DOI:10.1523/JNEUROSCI.5791-10.2011

Copyright $\odot 2011$ the authors $\quad 0270-6474 / 11 / 315540-09 \$ 15.00 / 0$ reaction, and redirecting attention back to the breath (Wallace, 2006; Lutz et al., 2008).

Meditation and pain both alter sensory, cognitive, and affective dimensions of our subjective experience (Koyama et al., 2005; Cahn and Polich, 2006). Given that mindfulness meditation can attenuate the subjective experience of pain (Grant and Rainville, 2009; Brown and Jones, 2010; Zeidan et al., 2010b), brain regions involved in meditation must somehow interact with those involved in nociceptive processing, yet the specific brain mechanisms associated with mindfulness meditation remain poorly characterized. In addition, how these brain mechanisms could potentially influence painrelated brain activity remains unknown (Buhle and Wager, 2010).

Using an emerging functional magnetic resonance imaging (fMRI) technique, pulsed arterial spin labeled (PASL) MRI, we investigated how meditation affects pain-related brain processes. PASL MRI is appropriate for imaging steady cognitive states such as meditation because it is less susceptible to slow drifts in signal intensity than conventional blood oxygen level-dependent (BOLD) fMRI (Detre and Wang, 2002; Wang et al., 2003). Moreover, this technique provides a fully quantitative measure of cerebral blood flow (CBF) and allows global CBF changes such as those produced by respiratory confounds to be assessed. In addition, PASL MRI can assess activation in the orbitofrontal cortex (OFC), an area contributing to affective regulation processes, more effectively than BOLD (Wang et al., 2003).

We postulated that, in addition to reducing psychophysical pain ratings, meditation would modulate brain regions associ- 


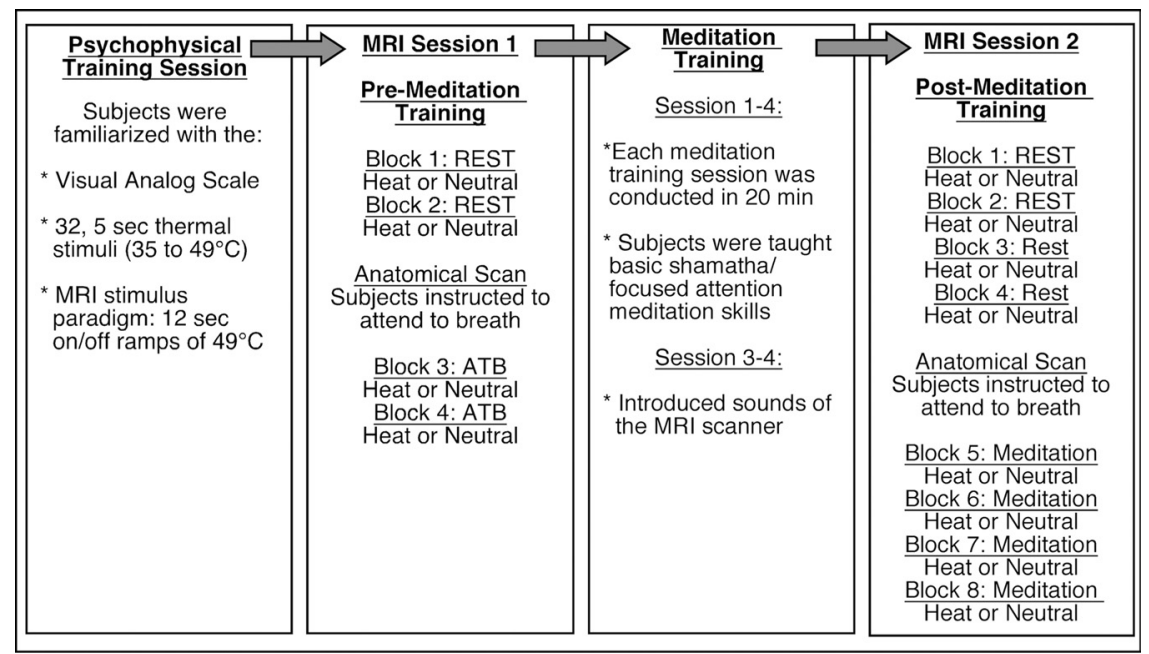

Figure 1. Experimental procedures across time. First column, Psychophysical training session: Subjects first came in for psychophysical training. In this session, subjects were familiarized with visual analog scales, the range of thermal stimulation, and thermal stimulation paradigm used in the subsequent MRI sessions. Second column, MRI session 1: In the first two blocks, subjects were asked to reduce movement and keep eyes closed (rest). A heat $\left(49^{\circ} \mathrm{C}\right)$ and neutral $\left(35^{\circ}\right)$ series were randomly presented in one of two blocks. Before anatomical acquisition, subjects were instructed to "begin meditating by focusing on the sensations of the breath." Subjects continued to attend to the breath during a block of noxious stimulation $\left(49^{\circ} \mathrm{C}\right)$ or neutral $\left(35^{\circ}\right)$. Pain ratings were assessed after each block. Third column: The $4 \mathrm{~d}(20 \mathrm{~min} / \mathrm{d})$ meditation intervention. Subjects were taught to focus on the changing sensations of the breath. They were taught that discursive thoughts were to be acknowledged without affective reaction and "let go" by redirecting their focus back on breath sensations. In sessions 3 and 4, sounds of the MRI scanner were introduced to familiarize subjects with the MRI environment. The fourth column describes the MRI session 2 (after meditation training). In the first four blocks, subjects were instructed to reduce movement and close their eyes (rest). Two heat $\left(49^{\circ} \mathrm{C}\right)$ and two neutral $\left(35^{\circ} \mathrm{C}\right)$ blocks were randomly administered. Before anatomical acquisition, subjects were instructed to "begin meditating by focusing on the sensations of the breath." Subjects continued to meditate across two blocks of noxious stimulation $\left(49^{\circ} \mathrm{C}\right)$ and neutral $\left(35^{\circ} \mathrm{C}\right)$. Pain ratings were assessed after each block.

ated with constructing the pain experience (Oshiro et al., 2009). We specifically hypothesized that meditation would engage brain regions such as the prefrontal cortex (PFC), anterior cingulate cortex (ACC), and anterior insula (AI), known for their role in attentional control and affective processing (Berthier et al., 1988; Raz and Buhle, 2006; Rolls and Grabenhorst, 2008). Furthermore, we sought to determine whether meditation-related activation of these brain regions was directly associated with pain modulation (Wiech et al., 2008).

\section{Materials and Methods}

Subjects. Eighteen subjects were recruited for participation in this study. During psychophysical training, one subject was dismissed for low sensitivity and one for hypersensitivity to heat stimulation. A third subject was dismissed for falling asleep during the course of the meditation intervention. Fifteen healthy volunteers, six males and nine females (age range, 22-35 years; mean age, 26 years), completed the study. All subjects were right-handed, 13 were Caucasian, one Asian, and one Hispanic. Subjects gave written, informed consent recognizing that (1) they would experience painful, heat stimuli, (2) all methods were clearly explained, and (3) they were free to withdraw from the study without prejudice. The Institutional Review Board of the Wake Forest University School of Medicine approved all procedures.

Overview of experimental procedures. An outline of experimental procedures is illustrated in Figure 1. Subjects participated in a psychophysical training session to familiarize them with the noxious stimuli and psychophysical rating procedures (see below, Psychophysical training). The first MRI session was conducted after psychophysical training but before meditation training (Fig. 1). In the first half of MRI session 1, subjects were instructed to close their eyes and reduce movement (rest condition). In the second half of both MRI sessions, subjects were instructed to "meditate by focusing on the changing sensations of the breath." In MRI session 1, this condition was labeled as "attention to breath" (ATB), thereby providing a control (i.e., divided attention) for comparisons with the meditation condition after training. Subjects then participated in $4 \mathrm{~d}$ of mindfulness meditation training (see below, Mindfulnessbased mental training). After successful completion of meditation training, subjects returned for MRI session 2. Similar to MRI session 1, in the first half of MRI session 2, subjects were instructed to close their eyes and reduce movement (rest condition). Afterward, subjects were instructed to "begin meditating by focusing on the changing sensations of the breath ["mindfulness-based attention to breath" (meditation)].

Psychophysical training. All subjects were initially familiarized with 32 5-s-duration stimuli $\left(35-49^{\circ} \mathrm{C}\right)$ to provide experience with the visual analog scales (VAS). After familiarizing subjects with the heat stimuli, they received 5 min and $55 \mathrm{~s}$ of stimulation, identical to the paradigm used in subsequent fMRI experimental sessions. A $15 \mathrm{~cm}$ plastic sliding VAS scale was used to quantify pain intensity and unpleasantness [Paresian Novelty (Price et al., 1994)]. The minimum rating was represented as "no pain sensation" or "not at all unpleasant," whereas the maximum was designated with "most intense imaginable" or "most unpleasant imaginable."

MRI session 1. Subjects were positioned in the MRI scanner, a pulse oximeter was attached to each subject's left index finger to assess heart rate, and a transducer was placed around the chest to gauge respiration rate. Noxious thermal stimuli were delivered to the posterior aspect of the right calf by a $16 \times 16 \mathrm{~mm}^{2}$ TSA II thermal stimulator (Medoc). The "heat" condition consisted of thermal stimuli that were administered in alternating patterns of heat $\left(49^{\circ} \mathrm{C}\right)$ and neutral $\left(35^{\circ} \mathrm{C}\right)$ with $12 \mathrm{~s}$ durations at each temperature $(5 \mathrm{~min}$ and $55 \mathrm{~s}$ total duration per MRI series). The baseline temperature was maintained at $35^{\circ} \mathrm{C}$, and stimulus temperatures were delivered with rise and fall rates of $6^{\circ} \mathrm{C} / \mathrm{s}$. The "neutral" series consisted of only neutral stimuli $\left(35^{\circ} \mathrm{C}\right.$ for 5 min and $55 \mathrm{~s}$ ). Thermal stimuli were counterbalanced across series. The heat probe was moved to a new location on the right calf after each series to reduce habituation. After each series, subject's evaluation of pain intensity and unpleasantness were acquired with the VAS scale. They were instructed that their ratings should reflect the overall experience of the entire series.

In MRI session 1, four functional series (two heat; two neutral) were separated by a structural acquisition scan. In the first half of the experiment, subjects were instructed to keep their eyes closed and restrict movement across conditions. After the structural image was obtained, subjects were instructed to "meditate by focusing on the changing sensations of the breath."

Mindfulness-based mental training. Mindfulness-based mental training was performed in four separate, 20 min sessions conducted by a facilitator with $>10$ years of experience leading similar meditation regimens. Subjects had no previous meditative experience and were informed that such training was secular and taught as the cognitive practice of Shamatha or mindfulness meditation. Each training session was held with one to three participants.

On mindfulness meditation training day 1 , subjects were encouraged to sit with a straight posture, eyes closed, and to focus on the changing sensations of the breath occurring at the tips of their nostrils. Instructions emphasized acknowledging discursive thoughts and feelings and to return their attention back to the breath sensation without judgment or emotional reaction whenever such discursive events occurred. On training day 2, participants continued to focus on breath-related nostril sen- 
sations and were instructed to "follow the breath," by mentally noting the rise and fall of the chest and abdomen. The last $10 \mathrm{~min}$ were held in silence so subjects could develop their meditative practice. On training day 3 , the same basic principles of the previous sessions were reiterated. However, an audio recording of MRI scanner sounds was introduced during the last $10 \mathrm{~min}$ of meditation to familiarize subjects with the sounds of the scanner. On the final training session (day 4), subjects received minimal meditation instruction but were required to lie in the supine position and meditate with the audio recording of the MRI sounds to simulate the scanner environment. Contrary to traditional mindfulness-based training programs, subjects were not required to practice outside of training.

Subjects also completed the Freiburg Mindfulness Inventory shortform (FMI), a 14-item assessment that measures levels of mindfulness, before psychophysical pain training and after MRI session 2. The FMI is a psychometrically validated instrument with high internal consistency (Cronbach $\alpha=0.86$ ) (Walach et al., 2006). Statements such as "I am open to the experience of the present moment" are rated on a five-point scale from 1 (rarely) to 5 (always). Higher scores indicate more skill with the mindfulness technique.

MRI session 2. After successful completion of meditation training, subjects participated in MRI session 2. This session consisted of eight functional series (four heat; four neutral). After completion of the first four "rest" series, subjects were again instructed to "meditate by focusing on the changing sensations of the breath," at which point the anatomical scan was conducted. Subjects were instructed to meditate until the end of the experiment. Four minutes after the anatomical scan, functional acquisition was resumed, and four meditation series were obtained (Fig. 1). Evaluation of pain ratings and experimental procedures were identical to MRI session 1.

MRI acquisition. CBF images were acquired on a 1.5 T GE Healthcare Twin-Speed LX Scanner with an eight-channel head coil (GE Healthcare) PASL MRI technique [Q2TIPS-FAIR (quantitative imaging of perfusion using a single subtraction, second version with interleaved thin-slice $\mathrm{TI}_{1}$ periodic saturation-flow-sensitive alternating inversion recovery)] (Luh et al., 1999). Scan parameters for the PASL Q2TIPS-FAIR acquisition are as follows: repetition time (TR), $2500 \mathrm{~ms}$; echo time (TE), $17.9 \mathrm{~ms}$; inversion time (TI), $1700 \mathrm{~ms}$; TI1, $700 \mathrm{~ms}$; TI2, $1200 \mathrm{~ms}$; field of view (FOV), $24 \times 18 \mathrm{~cm}$; matrix size, $64 \times 48$; slice thickness, $8 \mathrm{~mm}$; slice gap, $0 \mathrm{~mm}$; number of slices, 13; total number of volumes, 140 ; frequency direction, anteroposterior; total scan time, $5 \mathrm{~min} 55 \mathrm{~s}$. A two-dimensional echo planar imaging trajectory with ramp sampling was used for image acquisition. PASL is sensitive to subject motion, which may lead to inaccurate CBF maps. To remove the influence of subject motion on CBF quantification, the Q2TIPS-FAIR time series data (volumes 8-140) was filtered to remove individual perfusion weighted images that may corrupt the final CBF map (Tan et al., 2009). The first image in the PASL Q2TIPS-FAIR data was acquired with all inversion and saturation pulses turned off. This image was used as the M0 image to scale raw perfusion weighted images into a quantitative CBF map according to the general kinetic model (Buxton et al., 1998). Volumes 2-7 were needed to establish a steady-state prior and were not included when calculating the CBF maps.

Anatomical acquisition. After the rest condition, an accelerated $(2 \times)$ T1-weighted inversion recovery three-dimensional spoiled gradient echo structural scan was acquired (scan parameters: flip angle, $12^{\circ}$; TI, $600 \mathrm{~ms}$; resolution bandwidth, $15.6 \mathrm{kHz}$; FOV, $24 \times 24$; matrix size, $240 \times 240$; slice thickness, $1 \mathrm{~mm}$; number of slices, 164; acceleration factor, 2; total scan time, 3 min $57 \mathrm{~s}$ ).

Statistical analyses of regional signal changes within the brain. The functional image analysis package FSL [Functional Magnetic Resonance Imaging of the Brain (FMRIB) Software Library (Center for FMRIB, University of Oxford, Oxford, UK)] was used for image processing and analyses. Functional data were movement corrected and spatially smoothed with a $8 \mathrm{~mm}$ full-width at half-maximum three-dimensional isotropic Gaussian kernel. Each functional image was scaled by its mean global intensity (intensity normalization) to minimize confounds arising from global CBF fluctuations. All subject's functional images were registered to their structural data using a seven-parameter linear threedimensional transformation and then transformed into nonlinear
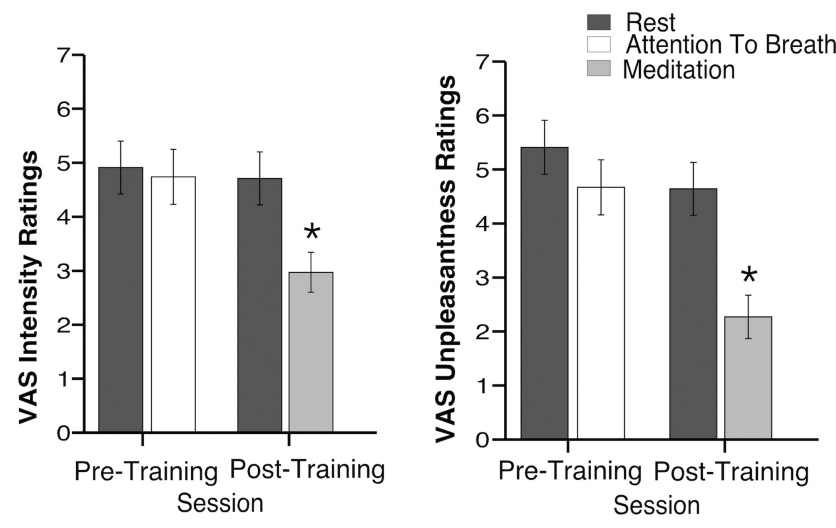

Figure 2. Mean (SEM) psychophysical pain ratings across each session. Meditation, after training significantly reduced pain intensity ratings and pain unpleasantness ratings when compared with rest ${ }^{*} p<0.05$.

standard stereotaxic space. High-resolution T1-weighted images were used to classify brain regions of activation and normalize images to standard space.

Statistical analysis of regional signal changes was performed on each acquisition series (first-level analyses) using a fixed-effects general linear modeling approach (Woolrich et al., 2001). Random effects analyses assessed activation across individuals. T/F statistic images were Gaussianized and thresholded using clusters determined by a $z$ $>2.3$. Corrected cluster significance threshold was set at $p<0.05$ (Worsley et al., 1992).

A two-way repeated-measures (RM) ANOVA was conducted on data from MRI session 1 to identify the main effects of pain and ATB. A similar RM ANOVA was conducted on data from MRI session 2 to identify main effects of pain and meditation, as well as their interaction. Paired comparisons were used to assess differences of main effects of pain and ATB/meditation between MRI sessions. These comparisons allowed us to differentiate brain mechanisms related to meditation (MRI session 2) from those associated with simple attention to breath (MRI session 1). To ensure that there was equal statistical power across sessions, only the first two volumes of the rest and meditation conditions in MRI session 2 were included to match the four volumes from MRI session 1 .

In MRI session 2, a multiple regression analysis using three regressors was conducted to assess the relationship between individual differences in meditation-induced pain reduction and brain activation. The first regressor was the mean effect of meditation versus rest in the presence of heat stimulation. Demeaned percentage changes for each subject's pain intensity ratings were entered as the second regressor, and demeaned percentage changes for each subject's unpleasantness ratings were entered as the third regressor. Intensity and unpleasantness regressors were orthogonalized to each other to identify variability in brain activity uniquely related to each aspect of pain.

Analysis of behavioral and physiological data. An RM ANOVA tested the effects of MRI session ( 1 and 2) between conditions (rest and ATB/meditation) on pain intensity and unpleasantness ratings (SPSS Inc.). We also examined FMI scores before and after mindfulness meditation training.

RM ANOVAs also tested the effects of rest, ATB, and meditation across neutral and heat stimulation conditions on heart rate, respiration rate, and global CBF across sessions (SPSS Inc.). Because of equipment malfunction, we used listwise deletion of six subjects for heart rate data and five subjects for respiration data in MRI session 1 (Allison, 2002).

\section{Results}

Pre-meditation training: MRI session 1 analyses

Attending to the breath does not reduce pain ratings

When subjects attended to their breathing before meditation training, no change in pain intensity $\left(F_{(1,14)}=0.92, p=0.35\right)$ or pain unpleasantness ratings $\left(F_{(1,14)}=4.31, p=0.06\right)($ Fig. 2$)$ was observed when compared with rest. 


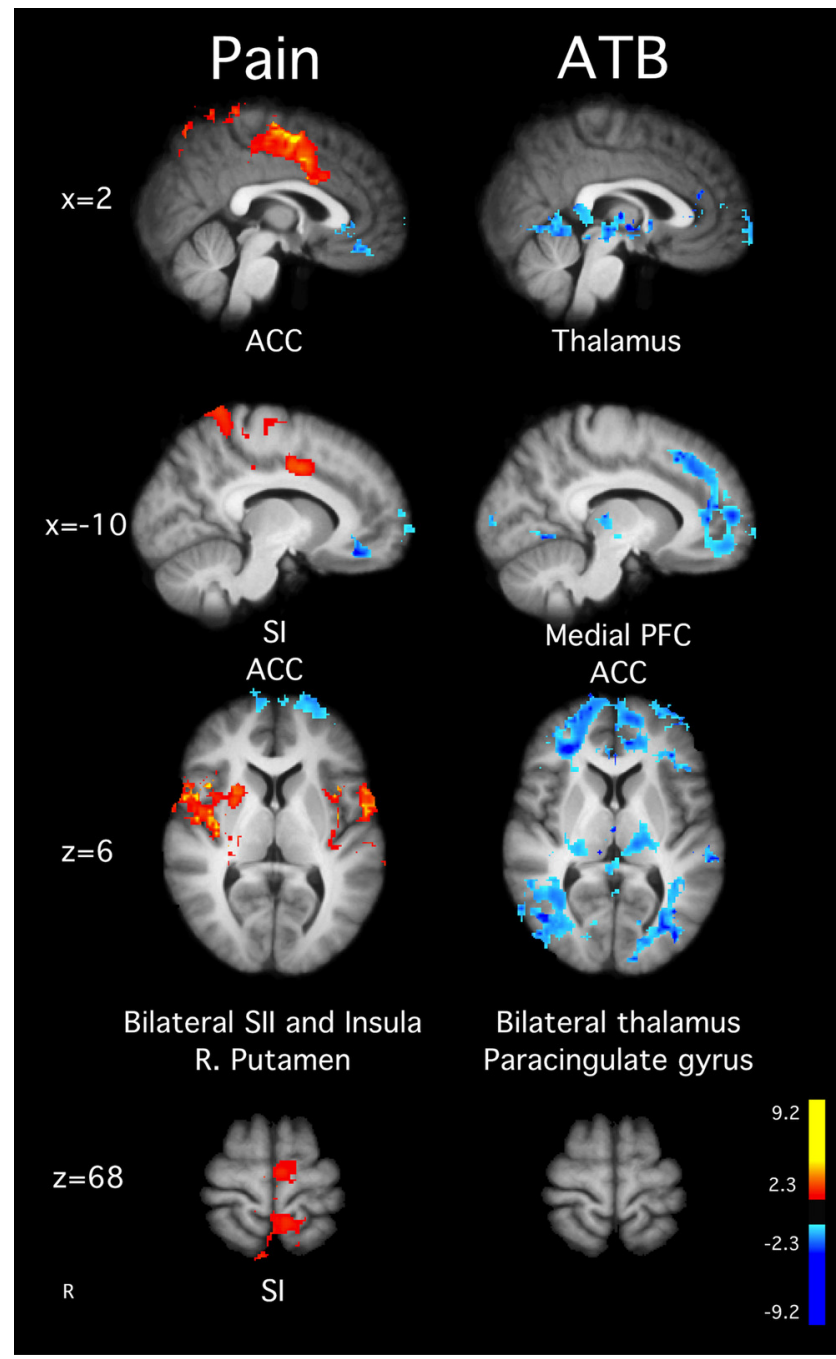

Figure 3. Brain activations and deactivations illustrate the main effects of ATB and pain in the MRI session before training. In the main effect of pain, there was greater activation in SI corresponding to the stimulation site, ACC, SII, left putamen, and bilateral insula. There was no ATB-related brain activity, but the deactivations for the main effect of meditation were found in the medial PFC, posterior cingulate cortex, thalamus, and paracingulate gyrus. Slice locations correspond to standard stereotaxic space.

Attention to breath-related brain activity

Before meditation training, there was no significantly greater brain activation when subjects were asked to focus on the sensations of the breath as contrasted to the rest condition (Fig. 3). However, there was significantly greater default-mode-related brain activation (medial PFC) in the rest condition (Raichle et al., 2001) compared with the ATB condition. These findings suggest that subjects were actively engaged in a cognitive task when instructed to focus on breath sensations than when compared with the rest condition. The thalamus, ACC, and paracingulate gyrus also were more activated in the rest condition.

Pain-related brain activity in MRI session 1

When compared with neutral stimulation, pain-related brain activity was detected in the ACC, bilateral insula, secondary somatosensory cortex (SII), and SI corresponding to the noxious thermal stimulation site on the leg (Fig. 3). These findings are consistent with previous studies using fully quantitative methods to examine CBF during pain (Coghill et al., 1999) and confirm that PASL methodology is sufficient to detect pain-related pat- terns of brain activation similar to those identified by BOLD fMRI or positron emission tomography (Owen et al., 2008).

Post-meditation training: MRI session 2 analyses

Brief meditation training improves mindfulness skills

The efficacy of meditative training was assessed with the FMI (Walach et al., 2006). Subjects exhibited a 14\% increase in mindfulness skills after $4 \mathrm{~d}$ of meditation training (mean $\pm \mathrm{SEM}$, $51.80 \pm 1.95$ ) compared with pretraining (mean \pm SEM, $45.33 \pm$ 2.00, $\left.F_{(1,14)}=11.68, p=0.004, \eta^{2}=0.46\right)$.

Meditation reduces pain intensity and unpleasantness ratings In MRI session 2, meditation produced a $40 \%$ reduction in VAS pain intensity ratings compared with rest (Fig. 2). Pain intensity ratings were lower in MRI session 2 compared with MRI session 1 (session main effect, $F_{(1,14)}=23.43, p<0.001, \eta^{2}=0.63$ ), and there was a significant main effect of condition (ATB/meditation vs rest, $\left.F_{(1,14)}=20.49, p<0.001, \eta^{2}=0.59\right)$. However, both main effects were driven by the substantial decrease in pain intensity ratings during meditation in MRI session 2 (interaction, $\left.F_{(1,14)}=14.23, p<0.001, \eta^{2}=0.50\right)$. Meditation also significantly reduced pain unpleasantness ratings by $57 \%$ $\left(F_{(1,14)}=87.99, p<0.001, \eta^{2}=0.86\right.$; main effect for session, $F_{(1,14)}=32.38, p<0.001, \eta^{2}=0.70$; condition, $F_{(1,14)}=$ 38.04, $p<0.001, \eta^{2}=0.73$; interaction, $F_{(1,14)}=18.55, p=$ 0.001, $\eta^{2}=0.57$ ) (Fig. 2).

\section{Meditation-related brain activity}

The form of mindfulness meditation (focused attention/ Shamatha) used relies on sustained attention to an explicit awareness of sensations associated with respiration with an emphasis of acknowledging discursive sensory events without affective reaction. Meditating, after training, produced bilateral activation of SI in regions corresponding to representations of the nose and throat (Penfield and Boldrey, 1937) as well as bilateral activation of the posterior insula and SII (Fig. 4). In addition, regions associated with interoceptive attention such as the AI and ACC also were bilaterally activated during meditation (Critchley, 2004). These regions, in addition to the OFC, ventral striatum, ventrolateral PFC, and amygdala also are associated with the cognitive modulation of pain (Rainville, 2002; Baliki et al., 2010). In contrast, significant posterior cingulate cortex and medial PFC deactivation was detected during meditation compared with rest (Fig. 4). This pattern of activity further distinguishes meditation from a resting default mode (Raichle et al., 2001; Farb et al., 2007).

\section{Pain-related brain activity in MRI session 2}

When compared with neutral stimulation, pain-related brain activation was identified bilaterally in the AI, posterior insula/ SII, ACC, and frontal operculum [Brodman's area (BA) 6/44] (Fig. 4).

\section{Meditation modulates pain-related brain processing}

The SI and supplementary motor area were sites of a significant interaction between meditation and noxious stimulation conditions. Post hoc analyses confirmed that, in the presence of noxious thermal stimuli, SI activation corresponding to the stimulation site (Penfield and Boldrey, 1937) significantly decreased during meditation when compared with rest (Fig. 5).

Diminished psychophysical pain ratings, however, also may have been influenced by meditation-induced activation of other regions important in pain processing. We found considerable overlapping activation in the AI between meditation and pain (Fig. 4). This region is activated during expectations for pain, 
placebo analgesia, and intensity evaluation (Wager et al., 2004; Koyama et al., 2005; Oshiro et al., 2009). Overlap between pain and meditation also was observed in mid-cingulate areas BA 24 and BA 32 (Fig. 4), areas associated with the processing of emotional contexts and cognitive control, respectively (Bush et al., 2000; Rainville, 2002; Vogt, 2005).

To better characterize brain regions associated with meditation-induced reductions in pain ratings, a regression analysis was conducted on the demeaned percentage decrease in VAS pain ratings between meditation and rest in the presence of noxious heat stimuli. In contrast to the previous within-subjects analyses, this analysis focused on identifying interindividual factors associated with the efficacy of meditation. Individuals with the greatest reductions in pain intensity ratings exhibited the largest meditationinduced activation of the right $\mathrm{AI}$ and bilateral ACC (Fig. 6). After accounting for the variance explained by brain mechanisms related to pain intensity reductions, individuals with the greatest reductions in pain unpleasantness ratings exhibited the greatest activation of the OFC and the greatest deactivation of the thalamus (Fig. 6).

Meditation activates areas related to selfregulation when compared with $A T B$ Meditating, after training, activated brain regions related to the self-regulation of pain. Areas involved in cognitive control (mid-ACC) (Vogt, 2005), emotion regulation (bilateral OFC) (Roy et al., 2009; Petrovic et al., 2010), and interoceptive awareness (right AI) (Critchley et al., 2004) (Fig. 7) were more active during meditation (after training) when compared with ATB (before training).

\section{Differences in the main effects of pain across MRI sessions}

The main effect of pain in MRI session 2 (collapsed across meditation and rest) was compared with the main effect of pain in MRI session 1 (collapsed across ATB and rest). After meditation training, reduced pain-related brain activation was observed in the leg representation of SI corresponding to the stimulation site (Penfield and Boldrey, 1937), SII, and posterior insula (Coghill et al., 1999, 2003) (Fig. 7). After meditation training, brain activity was more pronounced in the frontal pole, thalamus, medial PFC, and the ACC compared with before training.

\section{Global cerebral blood flow}

A three-way RM ANOVA ( session $\times$ stimulus $\times$ cognitive task) revealed a significant main effect of cognitive task on global CBF. There was a decrease in global CBF when subjects attended to the breath (i.e., ATB/meditation) (mean \pm SEM, 67.29 \pm 3.53 ) when compared with rest (mean $\pm \mathrm{SEM}, 70.26 \pm 2.93, F_{(1,14)}=5.38$, $p=0.04, \eta^{2}=0.28$ ) (Table 1 ). However, simple effects tests revealed no differences in global CBF between rest (mean \pm
SEM, $72.85+2.97)$ and ATB (mean \pm SEM, $70.09 \pm 3.32)$ in MRI session $1(p=0.09)$ or rest (mean \pm SEM, $67.68 \pm 2.88)$ and meditation (mean \pm SEM, $65.28 \pm 3.73$ ) in MRI session 2 $(p=0.16)$. There were no other significant main effects or interactions $(p$ values $>0.05)$.

\section{Respiration rate}

Respiration means (SEM) across conditions and sessions are reported in Table 1. Five subjects were not included because of equipment malfunction in MRI session 1. A three-way RM ANOVA (session $\times$ stimulus $\times$ cognitive task) revealed decreased respiration rate in MRI session 2 (mean \pm SEM, $13.72 \pm$ 0.79 ) compared with MRI session 1 (mean \pm SEM, $19.73 \pm 1.18$, $\left.F_{(1,9)}=36.81, p<0.001, \eta^{2}=0.80\right)$. Respiration rate was higher during heat (mean \pm SEM, $17.13 \pm 1.01$ ) than neutral stimulation $\left(\right.$ mean \pm SEM, $16.30 \pm 0.82, F_{(1,9)}=44.34, p<0.001, \eta^{2}=$ $0.83)$, and there were no differences between cognitive tasks $(p=0.19)$. Post hoc analyses revealed that the significant threeway interaction $\left(F_{(1,9)}=5.4, p<0.05, \eta^{2}=0.38\right)$ was indicative of a significant reduction in respiration rate during meditation in 


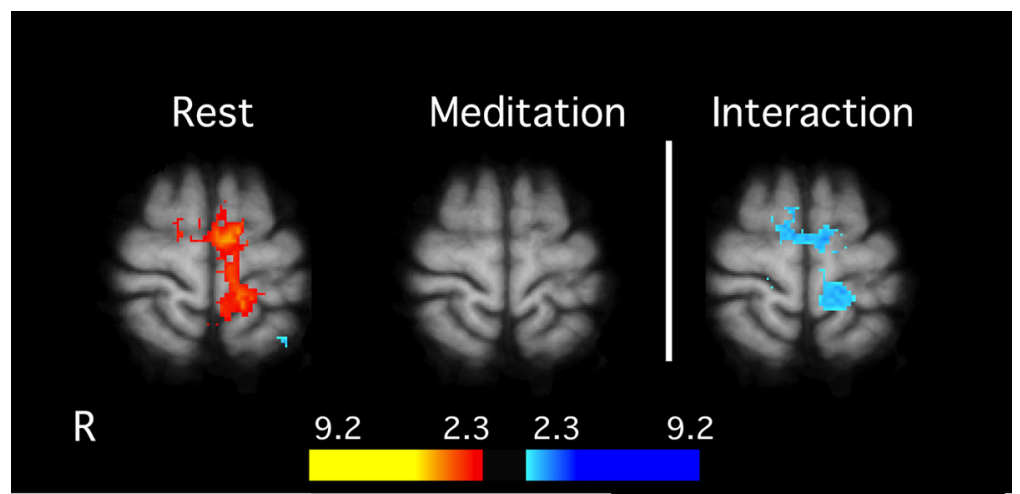

Figure 5. Interaction between meditation and pain-related brain activation in MRI session 2. General linear modeling analyses detected a significant interaction in SI $(z=68)$ between meditation and rest in the presence of noxious stimulation. There was a significant activation of the contralateral SI during heat stimulation while subjects were not meditating. Meditation significantly reduced activation in SI during noxious heat stimulation. Slice locations correspond to standard stereotaxic space.

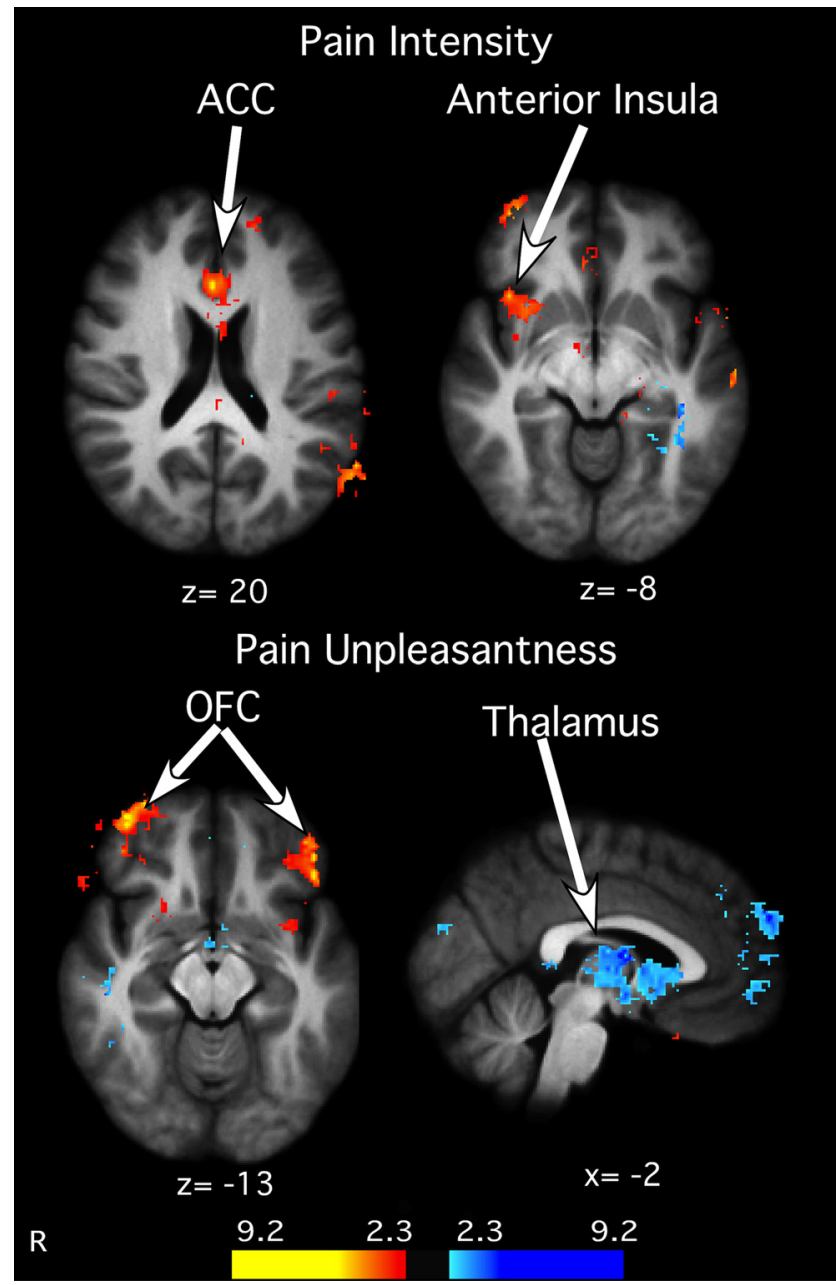

Figure 6. Relationship between meditation-induced decreases in psychophysical pain ratings and pain-related brain activation. Subjects reporting the greatest decrease in pain intensity ratings also exhibited the largest increase in the ACC and right anterior insula activation. Top, Similarly, subjects reporting the greatest activation in the OFC exhibited the greatest decreases in pain unpleasantness. In contrast, greater deactivation in the thalamus was related to larger decreases in pain unpleasantness ratings. Bottom, Slice locations correspond to standard stereotaxic space.

the presence of heat compared with all other conditions (Table 1). Decreases in respiration rate have been reported previously to predict reductions in pain ratings (Grant and Rainville, 2009;
Zautra et al., 2010). In the present data (MRI session 2; $n=14$ ), no significant relationship between the decreased respiration rates and pain intensity $(p=0.22$, $r=-0.35)$, pain unpleasantness $(p=$ $0.41, r=-0.24)$, or FMI ratings $(p=$ $0.42, r=0.24)$ was found.

\section{Heart rate}

Six subjects were not included because of equipment malfunction in MRI session 1 . A three-way RM ANOVA (session $\times$ stimulus $\times$ cognitive task) found that heart rate was higher during heat stimulation (mean \pm SEM, $75.18 \pm 2.56$ ) when compared with neutral stimulation $\left(\right.$ mean \pm SEM, $72.45 \pm 2.49, F_{(1,8)}=8.87$, $p=0.02, \eta^{2}=0.53$ ) (Table 1). There were no other significant differences or interactions ( $p$ values $>0.05$ ).

\section{Discussion}

For centuries, contemplatives have depicted meditation as a practice to rid oneself of mentality that "misconceives the inherent nature" of self (Nhấ and Vo-Dinh, 1987). In the present investigation, meditation reduced all subjects' pain intensity and unpleasantness ratings with decreases ranging from 11 to $70 \%$ and from 20 to $93 \%$, respectively. Moreover, meditation-related pain relief was directly related to brain regions associated with the cognitive modulation of pain. These findings provide unique insights into the manner that meditation alters the subjective experience of pain.

Meditation reduces pain through multiple brain mechanisms Meditation likely modulates pain through several mechanisms. First, brain areas not directly related to meditation exhibited altered responses to noxious thermal stimuli. Notably, meditation significantly reduced pain-related afferent processing in SI (Fig. 5), a region long associated with sensory-discriminative processing of nociceptive information (Coghill et al., 1999). Executivelevel brain regions (ACC, AI, OFC) are thought to influence SI activity via anatomical pathways traversing the SII, insular, and posterior parietal cortex (Mufson and Mesulam, 1982; Friedman et al., 1986; Vogt and Pandya, 1987). However, because meditation-induced changes in SI were not specifically correlated with reductions in either pain intensity or unpleasantness, this remote tuning may take place at a processing level before the differentiation of nociceptive information into subjective sensory experience.

Second, the magnitude of decreased pain intensity ratings was associated with ACC and right AI activation (Fig. 6). Activation in the mid-cingulate and AI overlapped between meditation and pain, indicating a likely substrate for pain modulation. Converging lines of evidence suggest that these regions play a major role in the evaluation of pain intensity and fine-tuning afferent processing in a context-relevant manner (Koyama et al., 2005; Oshiro et al., 2009; Starr et al., 2009). Such roles are consistent with the aspect of mindfulness meditation that involves reducing appraisals that normally impart significance to salient sensory events.

Third, OFC activation was associated with decreases in pain unpleasantness ratings (Fig. 6). The OFC has been implicated in 
regulating affective responses by manipulating the contextual evaluation of sensory events (Rolls and Grabenhorst, 2008) and processing reward value in the cognitive modulation of pain (Petrovic and Ingvar, 2002). Meditation directly improves mood (Zeidan et al., 2010a), and positive mood induction reduces pain ratings (Villemure and Bushnell, 2009). Therefore, meditation-related OFC activation may reflect altered executive-level reappraisals to consciously process reward and hedonic experiences (e.g., immediate pain relief, positive mood) (O'Doherty et al., 2001; Baliki et al., 2010; Peters and Büchel, 2010).

Meditation-related activation in these executive-level cortical areas may also influence thalamic nociceptive processing. The correlation between decreased pain unpleasantness ratings and the widespread deactivation of the thalamus during meditation may reflect a filtering mechanism associated with the modulation of ascending sensory information at thalamic levels (Fig. 6). The thalamic reticular nucleus (TRN) facilitates such filtering between frontal cortices and the thalamus (Crick, 1984). TRN is a GABAergic structure that operates as a "gatekeeper" between the thalamus and the cortex, in that all feedback connections between the thalamus and cortex travel through the TRN (Crick, 1984; Guillery et al., 1998). Projections from the PFC contact TRN GABAergic neurons, which in turn modulate sensory nuclei of the thalamus and thereby influence the transmission of subsequent sensory information to the cortex in a context-dependent manner (Sherman, 2001; Rauschecker et al., 2010). Cortically mediated shifts in executive attention activate TRN (Guillery et al., 1998; Kilmer, 2001), a mechanism fitting for sustaining attention and engaging/disengaging distractors (i.e., focused attention meditation). Thus, meditation-induced activation of this limbic-thalamic (Fig. 6) gating mechanism would modulate ascending noxious information before its accessing cortical regions implicated in conscious perception. The fact that TRN modulates visual and auditory modalities (Guillery et al., 1998) is consistent with the effects of meditation on binocular rivalry (Carter et al., 2005) and dichotic listening (Lutz et al., 2008). Together, these findings indicate that both corticocortical and corticothalamic interactions provide potential substrates for executive-order processes to alter the elaboration of nociceptive information into a subjectively available pain experience.

\section{Shared cognitive mechanisms for pain modulation}

Pain-relieving cognitive manipulations, such as attentional control, expectation manipulation, and placebo, all likely share common modulatory pathways (Wiech et al., 2008). We propose that meditation is another manipulation that also engages this final common pathway for pain modulation. For one, cognitive control is enhanced after training (Zeidan et al., 2010c), allowing
Table 1. Cerebral blood flow, respiration rate, and heart rate means (SEM) across conditions and sessions

\begin{tabular}{llll}
\hline & CBF & Respiration rate & Heart rate \\
\hline Session 1 & & & \\
$\quad$ Rest: neutral & $74.12(3.01)$ & $19.97(1.29)$ & $72.53(2.33)$ \\
$\quad$ Rest: heat & $71.51(2.93)$ & $20.45(1.11)$ & $74.79(2.39)$ \\
ATB: neutral & $70.69(3.56)$ & $17.05(1.00)$ & $70.46(1.79)$ \\
$\quad$ ATB: heat & $67.90(3.08)$ & $19.32(1.33)$ & $74.07(2.19)$ \\
Session 2 & & & \\
$\quad$ Rest: neutral & $68.57(3.17)$ & $16.72(0.82)$ & $74.82(3.08)$ \\
Rest: heat & $66.82(2.59)$ & $17.12(0.93)$ & $77.32(2.95)$ \\
$\quad$ Meditation: neutral & $65.09(3.59)$ & $11.55(0.74)$ & $73.62(2.77)$ \\
Meditation: heat & $65.47(3.86)$ & $9.47(0.67)^{a}$ & $75.38(2.70)$ \\
\hline
\end{tabular}

CBF was lower when subjects were instructed to attend to the breath $(n=15)$. However, there were no differences between rest and ATB in session 1 or between rest and meditation in session 2 .

${ }^{a}$ Respiration rate during heat stimulation and meditation was significantly lower than all other conditions $(n=10)$. Heat stimulation increased heart rate when compared with neutral stimulation $(n=9)$.

subjects to more effectively focus on the breath. This attentional set may reduce the saliency of noxious stimuli (Pessoa et al., 2003). Second, reducing expectations of impending noxious stimuli promotes pain modulation (Koyama et al., 2005). In meditation training, subjects were taught to reduce anticipation 
of future events, which may have lead subjects to reduce anticipatory responses to noxious stimuli (Brown and Jones, 2010). Finally, the notion that meditation could induce beliefs related to the promotion of pain relief is worth noting. Similar to previous reports examining placebo analgesia (Petrovic et al., 2002; Wager et al., 2004), the present study finds increased OFC and ACC activation related to pain modulation. However, meditation differs from placebo in that meditation is a volitionally induced cognitive state.

\section{Limitations}

The absence of significant differences in pain ratings during rest between MRI sessions 1 and 2 indicates that order effects related to the presentation of thermal stimuli, VAS usage, experimental instructions, scanner environment, and shifts in rating criteria were negligible. Although the within-group design controlled for these factors, the inclusion of a separate sham meditation control group may have provided additional insights into possible interactions between order effects and meditation training. For example, the inclusion of a sham meditation group would have allowed better assessment of demand characteristics related to the reputation of meditation. However, the relationship between executive-level brain regions and pain reductions indicates that neurocognitive factors other than report biases were engaged during meditation-induced reduction of pain reports.

One might also argue that the pain-relieving effects of meditation are simply attributable to divided attention rather than any unique attributes of mindfulness meditation per se. We believe that this explanation is unlikely because it fails to incorporate the differences in the mental processes involved between the two. For example, mindfulness meditation requires both the active allocation of attentional processes and the acknowledgment/regulation of responses to discursive stimuli. In contrast, divided attention likely lacks this meta-cognitive component. When compared to the divided attention condition, meditation was more effective at reducing pain ratings and evoking greater brain activation in areas involved in the cognitive/affective modulation of pain. Distraction has been found to reduce pain-related activity in the mid-ACC and AI (Bantick et al., 2002). In contrast, we found that increases in these areas were directly related to pain modulation. Moreover, in studies directly comparing meditation to distraction, meditation reduced pain ratings more than distraction (Zeidan et al., 2010b) and activated emotion regulatory brain regions (PFC, ACC) to a greater extent than distraction (Hölzel et al., 2007).

\section{Mindfulness meditation and pain}

Mindfulness meditation is characterized by two distinct cognitive practices. The fundamental practice of mindfulness is called focused attention (Lutz et al., 2008) or Shamatha (Sanskrit translation: calm abiding) (Wallace, 2006). Focused attention promotes a sense of detachment from ongoing affective states and enhances cognitive control (MacLean et al., 2010; Zeidan et al., 2010c). Traditionally, focused attention is cultivated as a prerequisite to another form of mindfulness meditation labeled open monitoring (Lutz et al., 2008) or Vipassana (Sanskrit translation: insight into the nature of reality) (Wallace, 2006). Open monitoring practitioners commonly refer to mindfulness as a moment-tomoment nonevaluative awareness of "whatever arises"(Wallace, 2006). The present findings, therefore, are distinct from the effects on pain by open monitoring. In open monitoring, meditators are taught to fully experience the intensity of a sensory event. Consistent with this, open monitoring has been found to reduce pain unpleasantness but not pain intensity ratings (Brown and Jones, 2010; Perlman et al., 2010). Thus, focused attention may attenuate pain by altering the elaboration of nociceptive information into pain, whereas open monitoring promotes a nonevaluative stance to a fully experienced sensory event.

Although the benefits of meditation have long been associated with extensive training (Grossman et al., 2004), our findings demonstrate that some of the beneficial effects of meditation can be realized after just $4 \mathrm{~d}(20 \mathrm{~min} / \mathrm{d})$ of training, suggesting that it may serve as an effective adjunct therapy in clinical settings. However, it is highly unlikely that the qualitative experience of meditation is similar across expertise level. In fact, studies have shown a positive relationship between meditative experience and pain relief (Brown and Jones, 2010; Grant et al., 2011), suggesting that the benefits of this technique can be further realized after extensive training. Nevertheless, we found that the increased capacity to reliably focus on the breath in a nonevaluative manner after brief training can effectively reduce the subjective experience of pain. Because meditation likely alters pain by enhancing cognitive control and reframing the contextual evaluation of nociceptive information, the constellation of interactions between expectations, emotions, and cognitive appraisals intrinsic to the construction of the sensory experience can be regulated by the meta-cognitive ability to nonjudgmentally sustain focus on the present moment.

\section{Notes}

Supplemental materials included are the brain coordinates for each analysis (available at www.jneurosci.org as supplemental material). This material has not been peer reviewed.

\section{References}

Allison PD (2002) Missing data. Thousand Oaks, CA: Sage Publications.

Baliki MN, Geha PY, Fields HL, Apkarian AV (2010) Predicting value of pain and analgesia: nucleus accumbens response to noxious stimuli changes in the presence of chronic pain. Neuron 66:149-160.

Bantick SJ, Wise RG, Ploghaus A, Clare S, Smith SM, Tracey I (2002) Imaging how attention modulates pain in humans using functional MRI. Brain 125:310-319.

Berthier M, Starkstein S, Leiguarda R (1988) Asymbolia for pain: a sensorylimbic disconnection syndrome. Ann Neurol 24:41-49.

Brown CA, Jones AK (2010) Meditation experience predicts less negative appraisal of pain: electrophysiological evidence for the involvement of anticipatory neural responses. Pain 150:428-438.

Buhle J, Wager TD (2010) Does meditation training lead to enduring changes in the anticipation and experience of pain? Pain 150:382-383.

Bush G, Luu P, Posner MI (2000) Cognitive and emotional influences in anterior cingulate cortex. Trends Cogn Sci 4:215-222.

Buxton RB, Wong EC, Frank LR (1998) Dynamics of blood flow and oxygenation changes during brain activation: the balloon model. Magn Reson Med 39:855-864.

Cahn BR, Polich J (2006) Meditation states and traits: EEG, ERP, and neuroimaging studies. Psychol Bull 132:180-211.

Carter OL, Presti DE, Callistemon C, Ungerer Y, Liu GB, Pettigrew JD (2005) Meditation alters perceptual rivalry in Tibetan Buddhist monks. Curr Biol 15:R412-R413.

Coghill RC, Sang CN, Maisog JM, Iadarola MJ (1999) Pain intensity processing within the human brain: a bilateral, distributed mechanism. J Neurophysiol 82:1934-1943.

Coghill RC, McHaffie JG, Yen YF (2003) Neural correlates of interindividual differences in the subjective experience of pain. Proc Natl Acad Sci U S A 100:8538-8542.

Crick F (1984) Function of the thalamic reticular complex: the searchlight hypothesis. Proc Natl Acad Sci U S A 81:4586-4590.

Critchley HD (2004) The human cortex responds to an interoceptive challenge. Proc Natl Acad Sci U S A 101:6333-6334.

Critchley HD, Wiens S, Rotshtein P, Ohman A, Dolan RJ (2004) Neural systems supporting interoceptive awareness. Nat Neurosci 7:189-195. 
Detre JA, Wang J (2002) Technical aspects and utility of fMRI using BOLD and ASL. Clin Neurophysiol 113:621-634.

Farb NA, Segal ZV, Mayberg H, Bean J, McKeon D, Fatima Z, Anderson AK (2007) Attending to the present: mindfulness meditation reveals distinct neural modes of self-reference. Soc Cogn Affect Neurosci 2:313-322.

Friedman DP, Murray EA, O’Neill JB, Mishkin M (1986) Cortical connections of the somatosensory fields of the lateral sulcus of macaques: evidence for a corticolimbic pathway for touch. J Comp Neurol 252:323-347.

Grant JA, Rainville P (2009) Pain sensitivity and analgesic effects of mindful states in Zen meditators: a cross-sectional study. Psychosom Med 71:106-114.

Grant JA, Courtemanche J, Rainville P (2011) A non-elaborative mental stance and decoupling of executive and pain-related cortices predicts low pain sensitivity in Zen meditators. Pain 152:150-156.

Grossman P, Niemann L, Schmidt S, Walach H (2004) Mindfulness-based stress reduction and health benefits. A meta-analysis. J Psychosom Res 57:35-43.

Guillery RW, Feig SL, Lozsádi DA (1998) Paying attention to the thalamic reticular nucleus. Trends Neurosci 21:28-32.

Hölzel BK, Ott U, Hempel H, Hackl A, Wolf K, Stark R, Vaitl D (2007) Differential engagement of anterior cingulate and adjacent medial frontal cortex in adept meditators and non-meditators. Neurosci Lett 421:16-21.

Kilmer W (2001) A thalamo-cortical model of the executive attention system. Biol Cybern 84:279-289.

Koyama T, McHaffie JG, Laurienti PJ, Coghill RC (2005) The subjective experience of pain: where expectations become reality. Proc Natl Acad Sci U S A 102:12950-12955.

Luh WM, Wong EC, Bandettini PA, Hyde JS (1999) QUIPSS II with thinslice TI1 periodic saturation: a method for improving accuracy of quantitative perfusion imaging using pulsed arterial spin labeling. Magn Reson Med 41:1246-1254.

Lutz A, Slagter HA, Dunne JD, Davidson RJ (2008) Attention regulation and monitoring in meditation. Trends Cogn Sci 12:163-169.

MacLean KA, Ferrer E, Aichele SR, Bridwell DA, Zanesco AP, Jacobs TL, King BG, Rosenberg EL, Sahdra BK, Shaver PR, Wallace BA, Mangun GR, Saron CD (2010) Intensive meditation training improves perceptual discrimination and sustained attention. Psychol Sci 21:829-839.

Mufson EJ, Mesulam MM (1982) Insula of the old world monkey. II. Afferent cortical input and comments on the claustrum. J Comp Neurol 212:23-37.

Nhấ H, Vo-Dinh M (1987) The miracle of mindfulness: a manual on meditation, Revised edition. Boston: Beacon.

O’Doherty J, Kringelbach ML, Rolls ET, Hornak J, Andrews C (2001) Abstract reward and punishment representations in the human orbitofrontal cortex. Nat Neurosci 4:95-102.

Oshiro Y, Quevedo AS, McHaffie JG, Kraft RA, Coghill RC (2009) Brain mechanisms supporting discrimination of sensory features of pain: a new model. J Neurosci 29:14924-14931.

Owen DG, Bureau Y, Thomas AW, Prato FS, St Lawrence KS (2008) Quantification of pain-induced changes in cerebral blood flow by perfusion MRI. Pain 136:85-96.

Penfield WB, Boldrey E (1937) Somatic motor and sensory representation in the cerebral cortex of man as studied by electrical stimulation. Brain 60:389-443.

Perlman DM, Salomons TV, Davidson RJ, Lutz A (2010) Differential effects on pain intensity and unpleasantness of two meditation practices. Emotion 10:65-71.

Pessoa L, Kastner S, Ungerleider LG (2003) Neuroimaging studies of attention: from modulation of sensory processing to top-down control. J Neurosci 23:3990-3998.

Peters J, Büchel C (2010) Neural representations of subjective reward value. Behav Brain Res 213:135-141.

Petrovic P, Ingvar M (2002) Imaging cognitive modulation of pain processing. Pain 95:1-5.

Petrovic P, Kalso E, Petersson KM, Ingvar M (2002) Placebo and opioid analgesia- imaging a shared neuronal network. Science 295:1737-1740.
Petrovic P, Kalso E, Petersson KM, Andersson J, Fransson P, Ingvar M (2010) A prefrontal non-opioid mechanism in placebo analgesia. Pain 150:59-165.

Price DD, Bush FM, Long S, Harkins SW (1994) A comparison of pain measurement characteristics of mechanical visual analogue and simple numerical rating scales. Pain 56:217-226.

Raichle ME, MacLeod AM, Snyder AZ, Powers WJ, Gusnard DA, Shulman GL (2001) A default mode of brain function. Proc Natl Acad Sci U S A 98:676-682.

Rainville P (2002) Brain mechanisms of pain affect and pain modulation. Curr Opin Neurobiol 12:195-204.

Rauschecker JP, Leaver AM, Mühlau M (2010) Tuning out the noise: limbic-auditory interactions in tinnitus. Neuron 66:819-826.

Raz A, Buhle J (2006) Typologies of attentional networks. Nat Rev Neurosci $7: 367-379$.

Rolls ET, Grabenhorst F (2008) The orbitofrontal cortex and beyond: from affect to decision-making. Prog Neurobiol 86:216-244.

Roy M, Piché M, Chen JI, Peretz I, Rainville P (2009) Cerebral and spinal modulation of pain by emotions. Proc Natl Acad Sci U S A 106: 20900-20905.

Sherman SM (2001) Tonic and burst firing: dual modes of thalamocortical relay. Trends Neurosci 24:122-126.

Starr CJ, Sawaki L, Wittenberg GF, Burdette JH, Oshiro Y, Quevedo AS, Coghill RC (2009) Roles of the insular cortex in the modulation of pain: insights from brain lesions. J Neurosci 29:2684-2694.

Tan H, Maldjian JA, Pollock JM, Burdette JH, Yang LY, Deibler AR, Kraft RA (2009) A fast, effective filtering method for improving clinical pulsed arterial spin labeling MRI. J Magn Reson Imaging 29:1134-1139.

Villemure C, Bushnell MC (2009) Mood influences supraspinal pain processing separately from attention. J Neurosci 29:705-715.

Vogt BA (2005) Pain and emotion interactions in subregions of the cingulate gyrus. Nat Rev Neurosci 6:533-544.

Vogt BA, Pandya DN (1987) Cingulate cortex of the rhesus monkey. II. Cortical afferents. J Comp Neurol 262:271-289.

Wager TD, Rilling JK, Smith EE, Sokolik A, Casey KL, Davidson RJ, Kosslyn SM, Rose RM, Cohen JD (2004) Placebo-induced changes in FMRI in the anticipation and experience of pain. Science 303:1162-1167.

Walach HB, Buttenmuller N, Kleinknecht V, Schmidt N (2006) Measuring mindfulness- the Freiburg Mindfulness Inventory (FMI). Pers Individ Dif 40:1543-1555.

Wallace BA (2006) The attention revolution: unlocking the power of the focused mind. Somerville, MA: Wisdom Publications.

Wang J, Aguirre GK, Kimberg DY, Roc AC, Li L, Detre JA (2003) Arterial spin labeling perfusion $\mathrm{fMRI}$ with very low task frequency. Magn Reson Med 49:796-802.

Wiech K, Ploner M, Tracey I (2008) Neurocognitive aspects of pain perception. Trends Cogn Sci 12:306-313.

Woolrich MW, Ripley BD, Brady M, Smith SM (2001) Temporal autocorrelation in univariate linear modeling of FMRI data. Neuroimage 14:1370-1386.

Worsley KJ, Evans AC, Marrett S, Neelin P (1992) A three-dimensional statistical analysis for $\mathrm{CBF}$ activation studies in human brain. J Cereb Blood Flow Metab 12:900-918.

Zautra AJ, Fasman R, Davis MC, Craig AD (2010) The effects of slow breathing on affective responses to pain stimuli: an experimental study. Pain 149:12-18

Zeidan F, Johnson SK, Gordon NS, Goolkasian P (2010a) Effects of brief and sham mindfulness meditation on mood and cardiovascular variables. J Altern Complement Med 16:867-873.

Zeidan F, Gordon NS, Merchant J, Goolkasian P (2010b) The effects of brief mindfulness meditation training on experimentally induced pain. J Pain 11:199-209.

Zeidan F, Johnson SK, Diamond BJ, David Z, Goolkasian P (2010c) Mindfulness meditation improves cognition: evidence of brief mental training. Conscious Cogn 19:597-605. 